\title{
Synchronous Clival Chordoma and Sellar-Suprasellar Ger- minoma in a Pediatric Patient: Case Report and Review of Literature
}

\author{
Vidur Bhalla ${ }^{1 *}$, Thomas Muelleman ${ }^{1}$, Chelsea S Hamill', D David Beahm ${ }^{1}$, Hesham M \\ Soliman ${ }^{2}$, Roukoz B Chamoun ${ }^{2}$ and Kathy Newell ${ }^{3}$
}

${ }^{1}$ Department of Otolaryngology, The University of Kansas, Kansas, USA

${ }^{2}$ Department of Neurosurgery, The University of Kansas, Kansas, USA

${ }^{3}$ Department of Pathology, The University of Kansas, Kansas, USA

*Corresponding author: Vidur Bhalla, MD, Department of Otolaryngology, The University of Kansas, Kansas City, Kansas, USA, Tel: 913-588-6732, Fax: 913-585-7415, E-mail: vbhalla@kumc.edu

\begin{abstract}
Pediatric brain tumors are the leading cause of cancer in children. While children more likely present with craniopharyngiomas, germinomas or optic gliomas, synchronous intracranial lesions are highly uncommon. Here, we describe the case of a 10-year-old female who presented with central Diabetes Insipidus (DI). MRI revealed and enhancing sellar/ suprasellar mass. The patient was taken to the operating room for endonasal resection. Pathology was consistent with sellar/suprasellar germinoma and contiguous clival chordoma. To the best of our knowledge this is the first reported case of association between these two rare tumors.
\end{abstract}

\section{Keywords}

Sellar/Suprasellar germinoma, Clival chordoma

\section{Introduction}

Though occurring at one-tenth the frequency of adult brain tumors, pediatric brain tumors are the leading cause of cancer related morbidity and mortality in children [1]. Synchronous intracranial lesions are highly unlikely, particularly those of separate, distinct cell types. Patients most at risk are those with neurocutaneous disorders, prior history of tumors, or history of chemotherapy and radiation.

Pediatric suprasellar tumors are distinct from their adult counterparts. While adults are more likely to have meningiomas or pituitary adenomas, children are more likely to present with craniopharyngiomas, germinomas, or optic gliomas [2]. Pathology determines appropriate treatment, and endonasal approaches, when appropriate; limit morbidity associated with surgery [3].

\section{Case Report}

A Ten-year-old female with history of cognitive delay, autism spectrum disorder and attention deficit hyperactivity disorder presented to her primary care provider with polydipsia, polyuria, enuresis, and 20 lb weight loss in the last year. Review of systems was positive for headache and daily vomiting. Past surgical history, social history, and family history were non-contributory. Vital parameters demonstrated tachycardia and hypertension, and the rest of her exam was unremarkable. Complete Blood Count (CBC) and urinalysis demonstrated no abnormalities. Complete Metabolic Panel (CMP) was significant for hypernatremia, hyperchloremia, and non-anion gap acidosis. Serum osmolality was high. The patient was directly admitted for work up of Diabetes Insipidus (DI), with renal, and endocrinology consults.

As an inpatient, ddAVP test demonstrated central etiology for her DI and hormonal work up showed panhypopituitarism. Subsequent MRI brain with and with- 


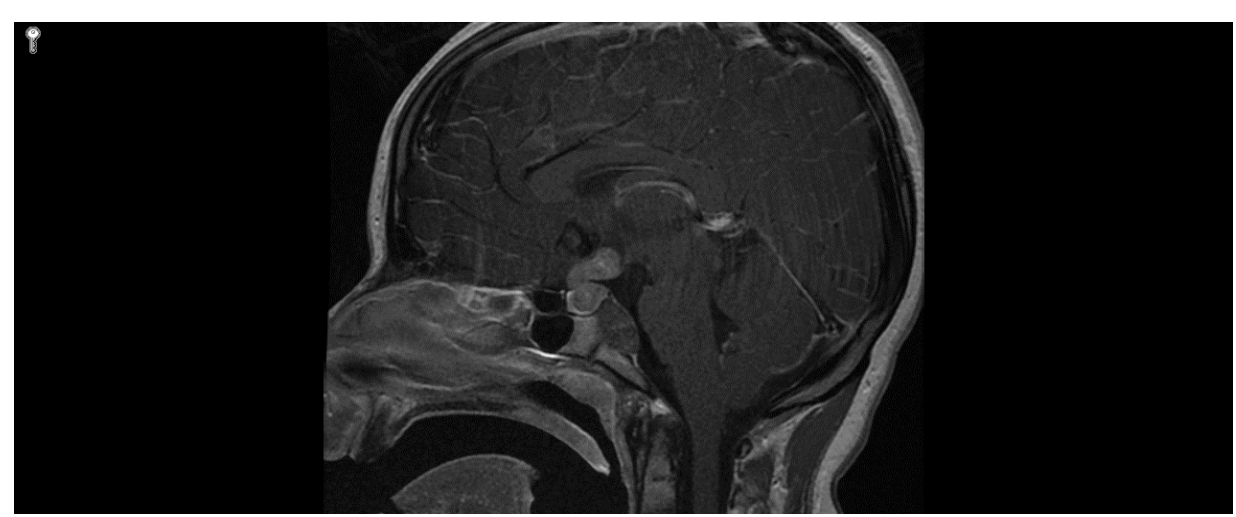

Figure 1: Preoperative MRI Head, T1 with contrast, sagittal view demonstrating an enhancing sellar/suprasellar mass with a possible cystic non-enhancing clival component measuring $2.2 \times 1.2 \times 1.7 \mathrm{~cm}$.

Figure 2: The clival lesion consists of cells with bubbly, vacuolated cytoplasm. These cells showed strong cytokeratin expression (not shown). (Hematoxylin and eosin, $\times 400$ original magnification).

out contrast revealed an enhancing sellar/suprasellar mass with a possible cystic non-enhancing clival component measuring $2.2 \times 1.2 \times 1.7 \mathrm{~cm}$ (Figure 1). Serum beta-HCG and alpha-FP were found to be within normal limits. CSF beta-HCG was 22 while CSF alpha-FP was < 0.5. Differential diagnosis included craniopharyngioma, germ cell tumor, atypical pituitary adenoma, or Langerhaan's histiocytosis. The possibility of two separate tumors was considered, although thought to be less likely. Ophthalmologic exam demonstrated no visual field defects or optic disc edema/pallor.

The patient was medically optimized and taken to the operating room by otolaryngology and neurosurgery for endoscopic endonasal, transsphenoidal approach and resection. A lumbar drain was placed prior to surgery. Intraoperatively, the sphenoid sinus was opened widely and the clivus was drilled between the carotids. An extradural, brown mass was noted and multiple biopsies demonstrated chordoma on pathologic section (Figure 2). Image guid- ance was used to aid in further surgery of this area and the tumor was resected without a Cerebrospinal Fluid (CSF) leak. The bone overlying the sellaturcica and suprasellar area at the level of the tuberculum sellae was removed. A thin portion of pituitary gland was noted, and this was dissected until a soft and friable tumor was encountered. The suprasellar dura was opened and the tumor was noted to be tracking toward the third ventricle, thus giving the tumor a classification of Type Illa [4]. Frozen and later permanent section was consistent with germinoma (Figure 3). Tumor was resected to the third ventricle, where visualization became more difficult. At this point, given the patient's age and pathology, the decision was made to conclude the resection. The patient's skull base defect was reconstructed with inlay and onlay Dura Matrix (Stryker Corporation, Kalamazoo, Michigan), anasoseptal flap, and resorbable packing.

Her lumbar drain was removed on Post-Operative Day (POD) 7 after no concern for CSF leak. Radiation 


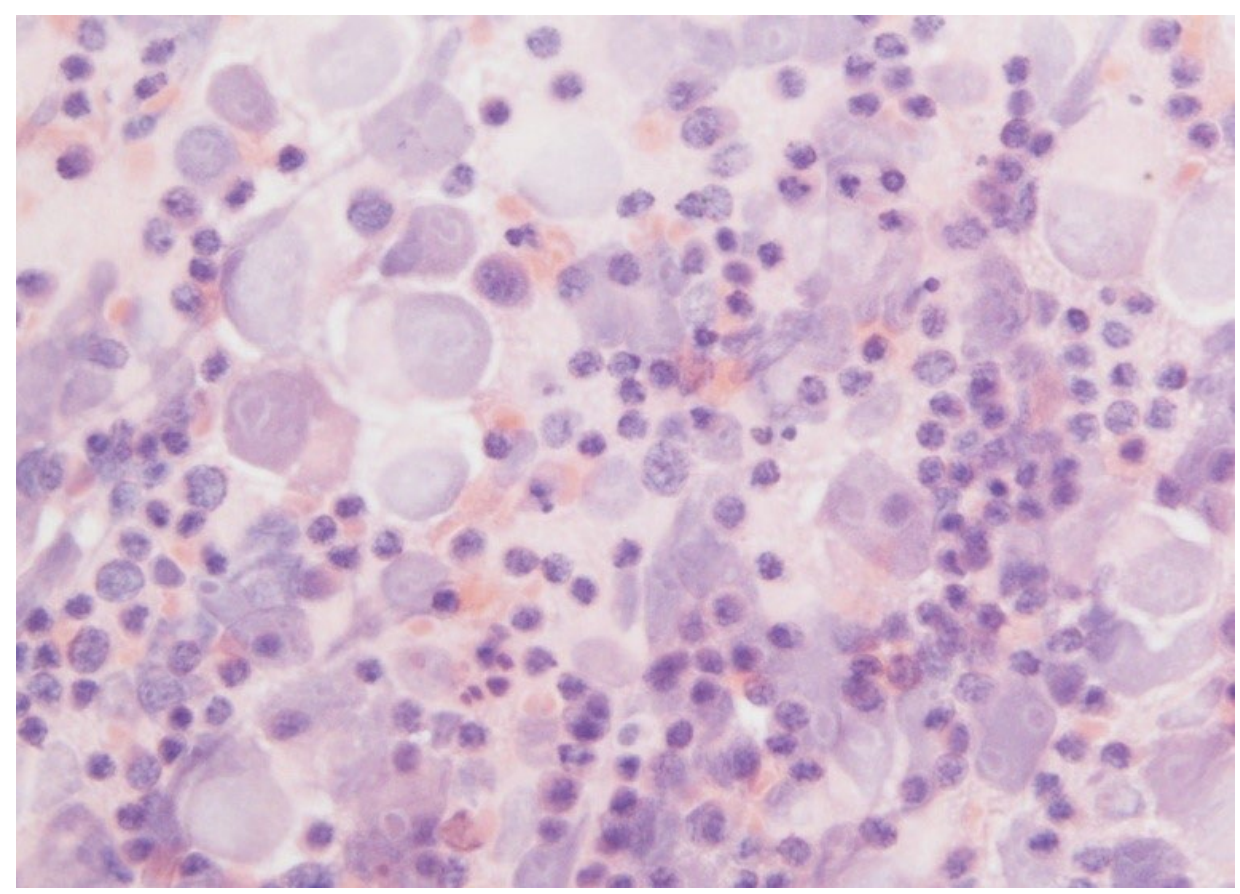

Figure 3: An intra-operative smear from the sellar/suprasellar mass confirms sheets of large atypical polygonal cells mixed with numerous small benign lymphocytes. (Hematoxylin and eosin, $\times 400$ original magnification).

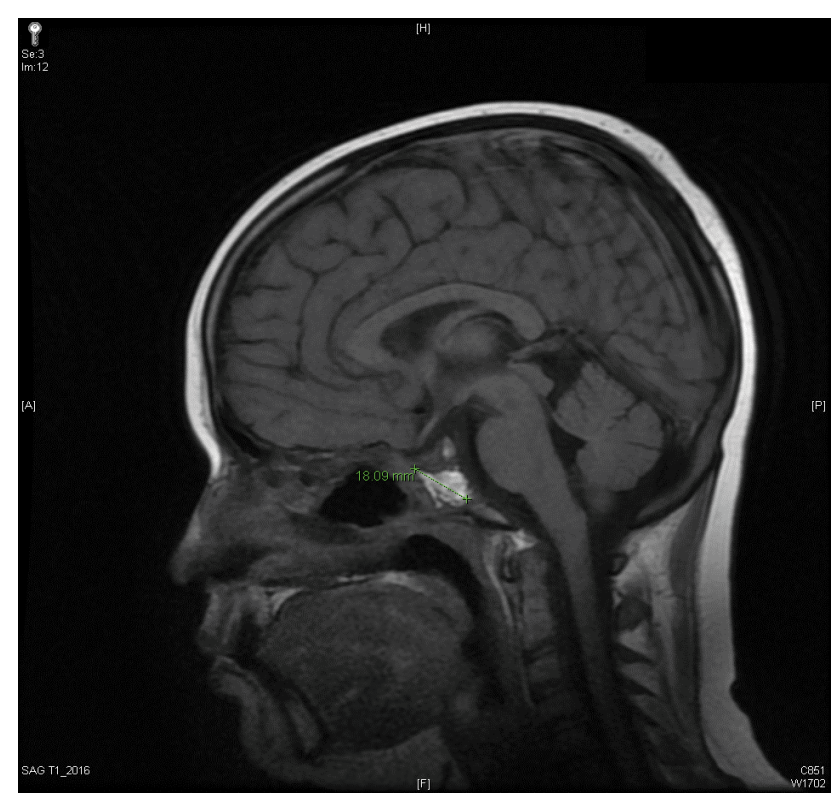

Figure 4: Postoperative MRI Head, T1 with contrast, sagittal view demonstrating fat packing of the clival operative cavity and no discrete residual or recurrent tumor identified.

oncology and hematology oncology teams were consulted and whole body positron emission tomography demonstrated no distant lesions. Radiation oncology recommended adjuvant radiation therapy, which was completed at an outside institution. The patient underwent cycles of chemotherapyfor 12 months after surgery (Figure 4).

Fourteen months after discharge, the patient presented with meningitis. Exam and imaging were concerning for CSF leak. The patient was taken to the OR for lumbar drain placement, fluorescein injection, and endoscopic repair of the leak via fat grafting in the sphe- noid sinus. Her drain was removed on POD 5, and there was no evidence of recurrent leak.

Twenty months after her first surgery, surveillance imaging demonstrated recurrence of her clivalchordoma. Revision endonasal endoscopic resection of the clival lesion was performed by extending the previous bony defect and further resection in this area. Postoperative recovery was uneventful.

Thirty-two months she again was reported to have a progressive enlargement of the clival mass on serial MRSIs. The patient's records were reviewed by an outside Proton Therapy Center and was determined to not be a candidate for proton beam radiation and surgery was recommended. A third surgery was performed at an outside institution thirty-five months after initial surgery. An abdominal fat graft was harvested for repair of the skull base defect and abdominal JP was left in place which was discontinued on POD2. On POD7 she was taken back for removal of the Merocels. Her postoperative course was complicated by worsening of her diabetes insibidus, requiring increasing her standing DDAVP dosing. She was discharged home on POD8.

\section{Discussion}

We discuss the case of a 10-year-old female with a sellar/suprasellar germinoma contiguous with a clivalchordoma. Germinomas represent $1 \%$ of all intracranial tumors, and of these, 20\% are suprasellar [5]. They typically develop from undifferentiated embryonal cells. These classically present with the triad of diabetes insipidus, panhypopituitarism, and visual changes [6]. Our patient did not exhibit the latter, however given her age and cognitive deficit, it is possible she could not articu- 
late these issues. Typically, treatment includes surgery, but these tumors are also chemo and radiation sensitive [7].

Chordomas develop at the ends of the notochord remnant near the sacrum and clivus. Although a predominance in the sacrum in the elderly, chordomas mostly affects the skull base in the pediatric population [8]. Though histologically low grade, they are locally invasive, highly recurrent and may metastasize [8]. Pediatric chordomas account for $5 \%$ of all chordomas, and typically present with atypical features and more aggressive pathology [8-10]. There are relatively low rates of complete resections and local recurrence may occur in up to $2 / 3^{\text {rds }}$ of patients [8]. As a result, post-operative radiation is a mainstay of therapy [8].

Both germinomas and chordomas are regarded as congenital or vestigial tumors. Whether clival or spinal in location, there has only been few reported cases in the literature of chordomas in association with other neoplasms. There was a report of a clivalchordoma found in association with a craniopharyngioma [11]. This was resected via open approach with resulting complete left cranial nerve III palsy. A more recent case, demonstrated sacrococcygeal chordoma synchronous with myxopapillary ependymoma identified on permanent section using immunochemistry [12]. To the best of our knowledge, this is the first reported case of association between chordoma and germinoma tumors. We suspect that because of the rarity of the two tumors, it would be unlikely that this is a pure coincidence. Further cytoanalysis or genetic analysis is needed to clarify the link between these two tumors.

\section{Conflicts}

There are no financial disclosures to be made.
This paper was presented as a poster at the North American Skull base Society meeting in February 2016.

\section{References}

1. Packer RJ (1999) Brain tumors in children. Arch Neurol 56 : 421-425.

2. Deopujari CE, Kumar A, Karmarkar VS, Biyani NK, Mhatre M, et al. (2011) Pediatric suprasellar lesions. J Pediatr Neurosci 6: S46-S55.

3. Yano S, Hide T, Shinojima N, Hasegawa $\mathrm{Y}$, Kawano T, et al. (2014) Endoscopic endonasal skull base approach for parasellar lesions: Initial experiences, results, efficacy, and complications. Surg Neurol Int 5: 51.

4. Kassam $A B$, Gardner PA, Snyderman $\mathrm{CH}$, Carrau RL, Mintz AH, et al. (2008) Expanded endonasal approach, a fully endoscopic transnasal approach for the resection of midline suprasellar craniopharyngiomas: a new classification based on the infundibulum. J Neurosurg 108: 715-728.

5. Jellinger K (1973) Primary intracranial germ cell tumours. Acta Neuropathol 25: 291-306.

6. Kageyama N, Belsky R (1961) Ectopic pinealoma in the chiasma region. Neurology 11: 318-327.

7. Leibel SA, Sheline GE (1987) Radiation therapy for neoplasms of the brain. J Neurosurg 66: 1-22.

8. Bilginer B, Turk CC, Narin F, Hanalioglu S, Oguz KK, et al. (2015) Enigmatic entity in childhood: clival chordoma from a tertiary center's perspective. Acta Neurochir (Wien) 157: 1587-1593.

9. Borba LA, Al-Mefty O, Mrak RE, Suen J (1996) Cranial chordomas in children and adolescents. J Neurosurg 84: 584-591.

10. Walcott BP, Nahed BV, Mohyeldin A, Coumans JV, Kahle KT, et al. (2012) Chordoma: current concepts, management, and future directions. Lancet Oncol 13: e69-e76.

11. Belza J (1966) Double midline intracranial tumors of vestigial origin: contiguous intrasellar chordoma and suprasellar craniopharyngioma. Case report. J Neurosurg 25: 199-204.

12. Vroobel K, Thway K (2016) Synchronous Sacrococcygeal Myxopapillary Ependymoma and Chordoma. Int J Surg Pathol 24: 48-50. 